\title{
Targeting SUMO-1ylation Contrasts Synaptic Dysfunction in a Mouse Model of Alzheimer's Disease
}

\author{
Serena Marcelli ${ }^{1}$ Elena Ficulle ${ }^{2} \cdot$ Filomena Iannuzzi $^{1} \cdot$ Enikö Kövari $^{3}$ • \\ Robert Nisticò $^{4,5}$ - Marco Feligioni ${ }^{1,2}$
}

Received: 13 May 2016/Accepted: 28 September 2016/Published online: 13 October 2016

(C) Springer Science+Business Media New York 2016

\begin{abstract}
Synaptic dysfunction has been recognized as an early feature occurring at the onset of Alzheimer's disease (AD). Compromised neurotransmission leads over time to synaptic loss and these events correlate with the cognitive decline that progressively affects $\mathrm{AD}$ patients.

Protein SUMOylation (Small Ubiquitin-like MOdifier) is a post-translational modification (PTM) involved in several cellular processes including synaptic transmission.

We here demonstrate that cortical synaptosomes prepared from $\operatorname{Tg} 2576$ mice of 6 months of age show an increased SUMO-1ylation, which returns back to normal levels at 20 months although synaptic SUMOylation, at this age, resulted more sensible to $\mathrm{KCl}$ stimulus. Our previous findings have shown that increased SUMOylation at presynaptic level reduces the $\mathrm{KCl}$-induced glutamate
\end{abstract}

Serena Marcelli and Elena Ficulle share co-first authorship.

Electronic supplementary material The online version of this article (doi:10.1007/s12035-016-0176-9) contains supplementary material, which is available to authorized users.

Marco Feligioni

m.feligioni@ebri.it

1 Laboratory of Neuronal Cell Signaling, EBRI Rita Levi-Montalcini Foundation, Rome, Italy

2 Department of Neurorehabilitation Sciences, Casa Cura Policlinico, Milan, Italy

3 Department of Psychiatry, University Hospitals of Geneva, Geneva, Switzerland

4 Pharmacology of Synaptic Disease, EBRI Rita Levi-Montalcini Foundation, Rome, Italy

5 University of Rome "Tor Vergata", Rome, Italy release. Accordingly, Tg2576 mice of 6 and 20 months show a reduced $\mathrm{KCl}$-evoked neurotransmitter (NT) release. In order to target SUMOylation, we developed two cell penetrating HIV Tat-linked peptides, namely TU-1 and TS-1. This strategy allowed us to modulate the SUMO machinery either positively (TU-1) or negatively (TS-1). As expected, Tg2576 synaptosomes treated with TU-1 exhibited a reduced NT release evoked by KCl. On the contrary, TS-1 treatment, which decreased SUMOylation, was able to normalize impaired glutamate release. Notably, an analysis of autopsy human AD brains has shown an increased SUMOylation in both cortical tissue and synaptosomal lysate. Our data indicate that SUMOylation level changes contribute to the development of synaptic alterations typically occurring at the $\mathrm{AD}$ onset and that SUMOylation could be a pharmacological target in AD synaptic dysfunction.

Keywords SUMO1 · Synapses · Neurotransmitter release · Alzheimer's $\cdot$ Human samples

\section{Introduction}

Alzheimer's disease (AD) is a progressive neurodegenerative condition characterized by the degeneration of nerve terminals which leads to a gradual, irreversible decline of cognitive functions, including memory loss. Intracellular neurofibrillary tangles and extracellular senile plaques are classical AD histopathological hallmarks, respectively composed of aggregating amyloid- $\beta$ peptides and hyperphosphorylated tau [1]. The severity of the pathology has been classified according to Braak staging, considering the accumulation of tau aggregates in the affected brain areas $[2,3]$. 
Remarkably, recent studies claimed "synaptopathy" as a putative culprit at the basis of $\mathrm{AD}$ [4].

Synaptic dysfunction seems to be one of the first neuronal defects at the onset of AD and is associated with a compromised neurotransmission that leads to synaptic loss and lately to neuronal death.

It is known that nanomolar to low micromolar concentrations of soluble $A \beta$ oligomers preferentially cluster at synapses, inducing synaptotoxicity and subsequent reduction in synaptic density, according to the so called "amyloid hypothesis" [5, 6]. Increasing evidence highlights the several molecular modifications occurring at synaptic level in early onset of disease. For example, the enhanced activity of synaptic caspase-3, a pro-apoptotic protease, seems to cause synaptic deficit in 3 month old Tg2576 transgenic mice, even before the appearance of amyloid plaques [7]. Caspase-3 expression is also increased in human $\mathrm{AD}$ brains [8].

In addition, a deregulated $\mathrm{Ca}^{2+}$ homeostasis has been associated with neuritic dystrophy and dendritic spine loss in response to $A \beta$ insult [9]. $\mathrm{Ca}^{2+}$ leakage from ER and other potential excitotoxic events are therefore probably responsible for the presynaptic dysfunction occurring at the very early stages of AD synaptopathy [5].

Similarly to other dysregulated post-translational modifications, the Small Ubiquitin-like MOdifier (SUMO) has a role in AD pathogenesis [10, 11]. Three major SUMO paralogues, namely, SUMO-1, SUMO-2, and SUMO-3 are expressed in mammalian cells and conjugate to target lysine via a hierarchical yet highly dynamic enzymatic cascade. The mature SUMO form is activated in an ATP-dependent pathway and is covalently attached to the SUMO-E1 activating enzyme. The complex is then transferred to the SUMO-E2 conjugating enzyme, Ubc9, which lastly transports SUMO onto the lysine residue of the target protein, generally by means of a specific SUMO-E3 ligase. The cycle is completed by the reversible removal of SUMO from its target substrates, operated by one of the SENP proteases [12].

SUMO conjugation can alter a wide variety of cellular events, including transcriptional regulation, signal transduction, protein aggregation and/or degradation, cell cycle regulation and DNA repair, intracellular trafficking, protein-protein interactions, and synaptic transmission [12-14]. Consequently, it is not surprising that aberrant SUMOylation has been correlated to the onset and/ or progression of several pathologies, including AD. Indeed, dysregulation of SUMOylation was observed in human post mortem AD brains, as well as in the $\mathrm{Tg} 2576$ transgenic $\mathrm{AD}$ mouse model [15]. Focusing on synapses, proteins belonging to both pre- and post-synaptic compartments such as MEF2 and GluR6 are also SUMOylated $[16,17]$ potentially impacting on $\mathrm{AD}$ pathology. In our previous work, furthermore, we found that pre-synaptic modulation of protein SUMOylation, induced by recombinant SUMO-1 or SENP1, was able to modulate synaptosomal glutamate release and calcium influx, thereby intervening in synaptic functionality [17]. Recently, the involvement of SUMO-1 in synaptic function, spine density, and memory has been confirmed by using a transgenic miceoverexpressing SUMO-1 exclusively in neurons. Synaptic overexpression of SUMO was related with an age-dependent reduction in basal synaptic transmission and impaired presynaptic function [18].

Overall, albeit lot of effort has been made to unveil the implication of SUMOylation in the cognitive impairment characterizing $\mathrm{AD}$ various unanswered questions with potential therapeutic entailments still exist.

Therefore, SUMOylation has been recently proposed as novel key regulator of learning and memory in both normal synaptic functioning and $\mathrm{AD}$ neuropathogenesis.

In fact, it was shown that the inhibition of specific SUMO2 conjugation actually impaired hippocampal-dependent learning and LTP; whereas, supplementing SUMOylation via the transduction of its $\mathrm{E} 2$ conjugating enzyme Ubc9 rescued $\mathrm{A} \beta$ induced deficits in learning and memory. In contrast to SUMO-2, minimal effects were obtained with the SUMO-1 paralog, possibly due to its slower dynamic capacity [15].

Targeting the SUMO machinery, therefore, could prove to be a novel pharmacological avenue in AD therapy.

Consequently, in this work we have developed and manufactured two cell penetrating HIV Tat-linked peptides, namely TU-1 and TS-1, able to finely modulate the SUMO machinery either positively (TU-1) or negatively (TS-1).

In line with our previous findings [17], here, we report that TS-1 is able to reduce synaptosomal protein SUMO-1ylation in both basal and stimulated conditions, enhancing synaptic neurotransmission via glutamate release and calcium influx. On the other hand, TU-1 increases basal SUMO-1ylation, thus impairing transmitter release. Interestingly, decreasing SUMOylation by means of TS- 1 is potentially able to restore synaptic function via the normalization of glutamate release.

\section{Results}

\section{Synaptic SUMOylation Level Changes in Tg2576 Mice}

Recently, it has been shown that cerebral cortex lysate from $\mathrm{Tg} 2576$ mice at around 6 months of age display a significant protein SUMO-1ylation increase, while at 17-months SUMO1ylation returns to control levels [19]. For this animal model, 6 months can be considered an early phase of the pathology, since mice do not show cognitive deficit, despite they do exhibit an overproduction of $A \beta$ soluble oligomers that appear to be toxic for synaptic function [7].

In order to investigate SUMO-1ylation changes at synaptic level during $\mathrm{AD}$ progression, cortical synaptosomes have been prepared from Tg2576 mice at 6 and 20 months. 
The SUMO-1ylation level has been measured by western blotting using a specific antibody. SUMO-1 immunoreactivity showed that Tg2576 mice at 6 months have a significant increased SUMOylation in cortical synapses (Fig. 1a-b: $\operatorname{Tg} 2576=1.44 \pm 0.16 ; p<0.01)$. On the other hand, according to our previous data [19], synaptic SUMO-1ylation from Tg2576 mice at 20 months does not differ from control (Fig. 1b-c: $\operatorname{Tg} 2576=1.15 \pm 0.16$ ).

\section{SUMOylation Level in Cortical Synaptosomes from 6-Months Tg2576 Mice}

SUMO-1 expression level has been analyzed by confocal microscopy to further confirm that SUMOylation is increased in $\mathrm{Tg} 2576$ mice. Cortical synaptosomes were prepared from 6 months Tg2576 mice and probed for SUMO-1 with a specific antibody.

Synaptosomes were conveniently visible upon white light both in control and in Tg2576 mice samples (Fig. 1e).

In line with western blots results, SUMO-1 immunoreactivity is definitely more intense in the Tg2576 synaptosomes rather than controls (Fig. 1f: $1.33 \pm 0.11$ vs. control. ${ }^{*} p<0.05$ ). Accordingly, synaptosomes in control image are less bright than $\mathrm{Tg} 2576$, and the background signal in control is higher than in $\mathrm{Tg} 2576$ (Fig. 1e).

\section{Stimulated Exocytosis Recorded by Time-Lapse Imaging from Synaptosomes}

Neurotransmitter exocytosis has been studied through video time-lapse recording of the Synaptogreen fluorescent dye as already reported [20]. Synaptogreen, also known as FM1-43, is a fluorescent dye able to concentrate in presynaptic vesicles and be released after a depolarizing stimulation, while losing its fluorescence. Therefore, by recording time-lapse images, it is possible to measure synaptosomal exocytosis kinetics associated with the run-down of the fluorescence after a stimulus. During image analyses, responding synaptosomes were identified and the fluorescence changes of synaptic puncta were measured throughout the video.

Synaptosomes prepared from cortex of control and Tg2576 mice, respectively, of 6 and 20 months, were both treated with $30 \mathrm{mM}$ of $\mathrm{KCl}$. As previously reported, administration of $30 \mathrm{mM}$ of $\mathrm{KCl}$ is able to induce a reduction in fluorescence that corresponds to NT release [20].

Synaptosomes from $\mathrm{Tg} 2576$ mice of 6 months presented a reduced release of $\mathrm{NT}$ in response to $\mathrm{KCl}$ with respect to control (Fig. 2a-b: $30.58 \pm 5.64$ vs $20.06 \pm 5.75$; $* * * p<0.001)$. This result is in line with the evidence that Tg2576 mice at 6 months display a higher synaptic SUMOylation level (Fig. 1a-b) and with the previous observations that higher presynaptic SUMOylation is related to a reduction of neurotransmitter release [17].
Surprisingly, also synaptosomes from $\mathrm{Tg} 2576$ mice of 20 months showed a reduced NT release under $\mathrm{KCl}$ stimulus with respect to control (Fig. $2 \mathrm{c}-\mathrm{d}$ : $18.69 \pm 0.59$ vs $13.33 \pm 0.71 ; * * * p<0.001)$. This result let us to hypothesize that, although synaptosomes prepared from old transgenic mice do not show different presynaptic SUMOylation with respect to controls (Fig. 1c), it might be that SUMO conjugation machinery could respond differently during $\mathrm{KCl}$ depolarizing conditions.

\section{Synaptic SUMOylation Is Increased in Old Tg2576 Mice versus Control under Depolarizing Conditions}

To test our hypothesis, 20 months Tg2576 synaptosomes and their age-matched controls were stimulated with $\mathrm{KCl} 30 \mathrm{mM}$ for $90 \mathrm{~s}$ and then analyzed by western blotting. As already reported, $\mathrm{KCl}$ is an efficient stimulus to induce SUMOylation. Indeed, the increment of conjugated proteins can be obtained in SH-SY5Y cells by $50 \mathrm{mM} \mathrm{KCl}$ stimulus and it seems to be partially dependent on $\mathrm{Ca}^{2+}$ influx [21]. In addition, a stimulus with $30 \mathrm{mM} \mathrm{KCl}$ for $90 \mathrm{~s}$ is sufficient to augment SUMO protein conjugation in synaptosomes [17].

In accordance with these evidences, cortical synaptosomes from 20 months $\mathrm{Tg} 2576$ were therefore treated with $30 \mathrm{mM}$ $\mathrm{KCl}$ and compared to controls. In both cases, $\mathrm{KCl}$ was able to induce a significant increase in synaptic SUMOylation (Fig. 3a-b: control basal conditions $=1.00 \pm 0.09$ vs $\mathrm{KCl}=1.50 \pm 0.05 ; * p<0.05 ; \mathrm{Tg} 2576$ basal conditions $=1.13 \pm 0.05$ vs $\mathrm{Tg} 2576+\mathrm{KCl}=1.98 \pm 0.15 ; * * p<0.01$ ).

Surprisingly, Tg2576 synaptosomes showed an increased level of SUMOylation after stimulus compared to their respective control under same conditions (Fig. 3a-b: control + $\mathrm{KCl}=1.50 \pm 0.05$ vs $\mathrm{Tg} 2576+\mathrm{KCl}=1.98 \pm 0.15 ;$ \# $p>0.05$ ) while SUMOylation between control and Tg2576 in basal conditions resulted to be not significantly different (Fig. 3a-b: control basal condition $=1,00 \pm 0,09$ vs $\operatorname{Tg} 2576$ basal condition $=1,13 \pm 0,05$ ). This result suggests that, although brain lysate [19] and synaptic protein SUMOylation are not different in the older Tg2576 mice (Fig. 1c), synaptic protein conjugation level might be more inducible by depolarizing conditions during later stages of $\mathrm{AD}$.

\section{SUMOylation Level can Be Manipulated via Transduction of Ubc9 or SENP1}

Nowadays, only unspecific SUMO-targeted compounds, like ginkgolic acid or anacardic acid, are commercially available. In order to manipulate SUMOylation and to study its potential involvement in synaptic function, we decided to develop new SUMO-targeted tools by synthesizing fusion proteins combining the Tat protein transduction sequence, which confers high cell permeability, with an effector sequence. The HIV-1 Tat protein cysteine-rich domain has been previously used to successfully 

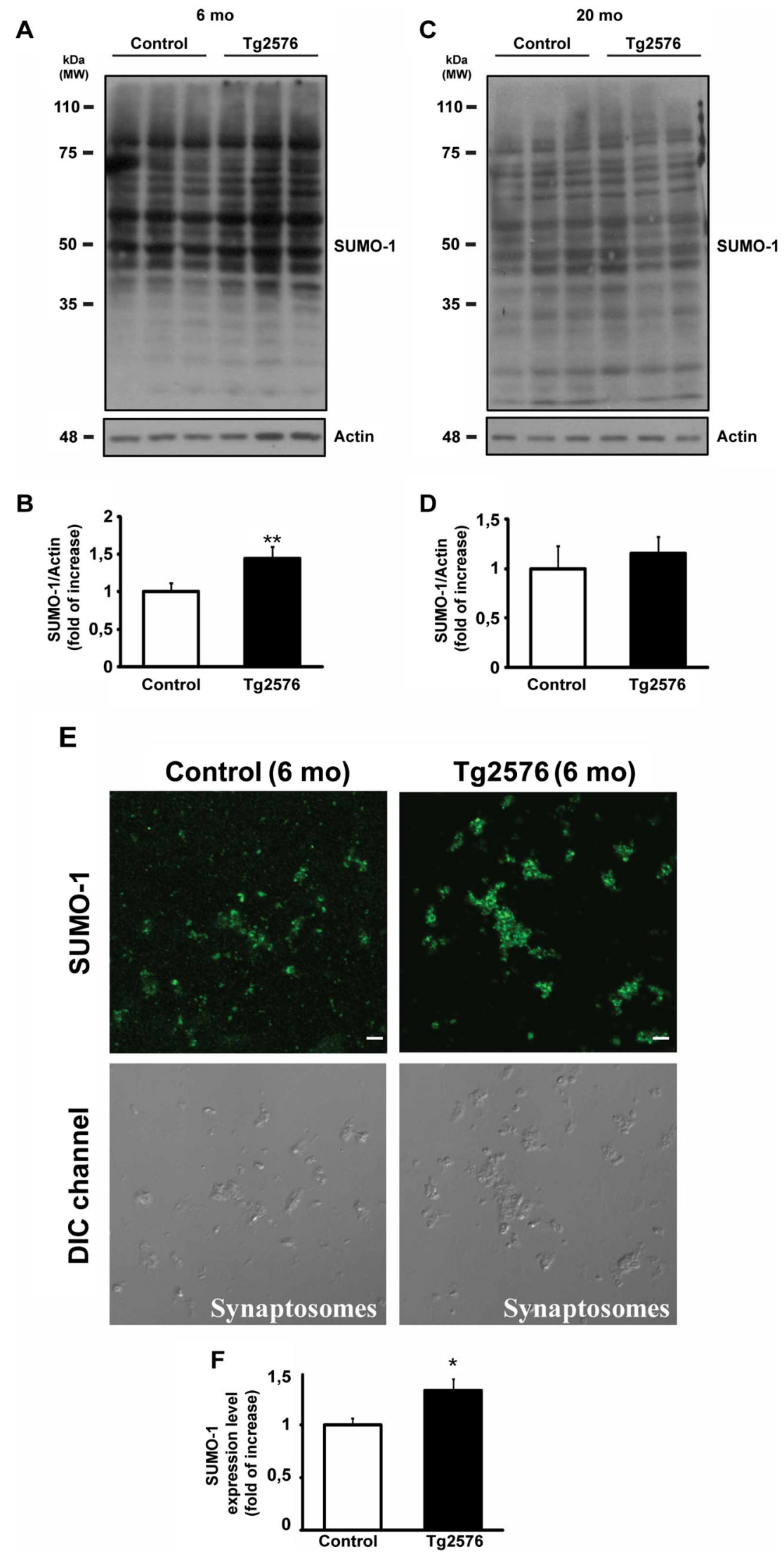
Fig. 1 SUMO-1 expression level in Tg2576 at 6 and 20 months of age .a Representative western blot of SUMO-1 expression level. Cortical synaptosomes from $\mathrm{Tg} 2576$ mice at 6 months and age-matched controls were lysed and used to run western blot. SUMO-1 antibody immunoreactivity showed that protein conjugation level is enhanced in $\mathrm{AD}$ mice compared to controls. b Quantification of SUMO-1 expression level at 6 months. SUMO-1 bands signal has been normalized versus actin. Results are expressed as fold of increase vs. the SUMO-1ylation level of control. Means \pm s.e.m. $n=3$ experiments run in triplicate, $* * p<0.01$ vs. control, $t$ test. c Representative western blot of SUMO-1 expression level. Cortical synaptosomes from $\mathrm{Tg} 2576$ mice at 20 months and age-matched controls were lysed and used to run western blot. SUMO-1 antibody immunoreactivity shows that protein conjugation level in $\mathrm{AD}$ mice does not differ from controls. d Quantification of SUMO-1 expression level at 20 months. SUMO-1 bands signal has been normalized versus actin. Results are expressed as fold of increase vs. the SUMO-1ylation level of control. Means \pm s.e.m. $n=3$ experiments run in triplicate, $t$ test has been performed but data do not differ significantly. e Representative confocal images (SUMO-1, green channel) and transmitted light (DIC channel) view of SUMO-1 staining. Scale bar $=10 \mu \mathrm{m}$. f Histogram showing the fold of increase of SUMO-1 expression level in Tg2576 synaptosomes at 6 months compared to age-matched controls. Means \pm s.e.m. $n=4$ experiments, ${ }^{*} p<0.05$ vs. control, $t$ test

deliver a variety of functional proteins as cell permeable peptides (CPPs) into cells $[22,23]$. Ubc9 is the solely known SUMO conjugating enzyme, while SENP1 is a specific SUMO isopeptidase enzyme. When Ubc9 is expressed in cell lines, it can induce SUMOylation, while SENP1 reduces it [12, 14, 24].

For our purposes, two plasmids have been engineered and a schematic representation of them is shown in Fig. 3c. TatUbc9 (TU-1; SUMOylation enhancer) and Tat-SENP1 (TS1; deSUMOylation inducer) proteins have been obtained by bacterial synthesis. Tat-SENP1 construct only expresses the functional core (351-644 amino acid residues) of the enzyme since the whole protein would have been too wide to be synthesized.

SENP1 core sequence is known to efficiently reduce protein SUMOylation when overexpressed in SH-SY5Y cells [24].

\section{Cell Permeable Peptides Modulate SUMOylation Level in a Neuroblastoma Cell Line}

TS-1 has been applied on a culture of neuroblastoma cells (SH-SY5Y) at two concentrations, $1 \mu \mathrm{M}$ and $0.5 \mu \mathrm{M}$, for $1.5 \mathrm{~h}$ before lysate preparation (Fig. 4a). Western blot analysis of the cell lysate showed that at both concentrations, TS-1 was able to reduce basal level of SUMOylation (Fig. $4 \mathrm{a}-\mathrm{c}: 0.58 \pm 0.06 ; * * * p<0.001$ (TS-1 $1 \mu \mathrm{M})$ and $0.46 \pm 0.09$; $* * * p<0.001$ (TS-1 $0.5 \mu \mathrm{M}$ ). Since no meaningful statistical difference has been found in the deSUMOylation effect of $0.5 \mu \mathrm{M}$ TS- 1 compared to $1 \mu \mathrm{M}$, the lower concentration $(0.5 \mu \mathrm{M})$ has been chosen for further experiments.
TU-1 was reported to be active already at $0 . \mu \mathrm{M}$ to induce an increase in SUMOylation but not able to increase basal level of SUMO-1ylation [15]. SH-SY5Y cells were treated with TU-1, applied for $1.5 \mathrm{~h}$ before harvest cells, in basal and $\mathrm{KCl}$ stimulated conditions. In line with the previous report, our data show that $\mathrm{KCl} 60 \mathrm{mM}$ significantly increased SUMO-1ylation (Fig. 4b-d: $1.38 \pm 0.10$; **p $<0.01$ ), but conversely and surprisingly $0.1 \mu \mathrm{M}$ of TU-1 induced a significant augment of SUMO-1ylation during basal condition (Fig. 4b-d: $1.51 \pm 0.06 ; * * p<0.01$ ). Apparently, our data are in contrast with the fact that TU-1 was not able to induce SUMO-1ylation increase, as found in [15]. Anyway, it has to be highlighted that our experiments were performed on cultured cells which are a different system than hippocampal slices. Moreover, when TU-1 was applied together with $\mathrm{KCl}$, SUMOylation was still increased although the expected synergic effect of $\mathrm{KCl}$ and TU-1 application was not observed (Fig. $4 \mathrm{~b}-\mathrm{d}: 1.44 \pm 0.14 ; * * p<0.01$ ). It is possible that the depolarizing effect is sufficient to reach the SUMOylation 'plateau', abolishing the effect of TU-1 that is not able to recruit additional conjugable SUMO proteins.

\section{TS-1 and TU-1 Modulate SUMOylation Levels in Cortical Synaptosomes}

The two peptides have been then tested by pre-treating for 30 min synaptosomes prepared from 6-months wild type mice, subsequently stimulated by $\mathrm{KCl}$ depolarization.

The synaptic level of SUMO has been efficiently modulated by the CPPs tested.

In fact, as it was already reported [17], $90 \mathrm{~s}$ of $30 \mathrm{mM}$ of $\mathrm{KCl}$ was able to induce an increased level of SUMOylation (Fig. 4e-g: $1.24 \pm 0.04 ; * * p<0.01$ ) which was reversed by application of $0.5 \mu \mathrm{M}$ TS-1. Indeed, TS1 significantly reduced the level of SUMOylation in both basal level and depolarizing conditions, although no differences between TS-1 treated synaptosomes in basal and $\mathrm{KCl}$ conditions were detected (Fig. $4 \mathrm{e}-\mathrm{g}$ : $0.68 \pm 0.09$; $* p<0.05$ (TS-1) and $0.57 \pm 0.09 ; * p<0.05$ (TS- $1+$ $\mathrm{KCl}$ ). This result indicates that probably, in this system, the deSUMOylation effect of TS-1 is so efficient that it can prevent the inducible SUMO conjugation induced by $\mathrm{KCl}$ stimulus.

Regarding the western blot of the TU-1 treatment, the effect of $\mathrm{KCl}$ on synaptosomes was again found efficient to increase SUMOylation (Fig. $4 \mathrm{f}-\mathrm{h}$ : $1.22 \pm 0.06$; $* p<0.05)$. Synaptosomes treated with TU-1 showed a significant augmented basal level of SUMO conjugation (Fig. 4f-h: $1.81 \pm 0.21 ; * * * p<0.001$ ). Intriguingly, TU-1 together with $\mathrm{KCl}$ induced a synergic increase of synaptic SUMO conjugation which was even higher than the $\mathrm{KCl}$ stimulus alone (Fig. 4f-h: $1.60 \pm 0.14$; ***p $<0.001$ ). This result suggests that depolarizing conditions are not 
Fig. 2 Tg2576 mice have a reduced $\mathrm{KCl}$-evoked exocytosis at both 6 and 20 months. Representative images of Synaptogreen (FM 1-43) stained cortical synaptosomes stimulated with $\mathrm{KCl}$ and recorded for 30 min: control of 6 months (a, 1), Tg2576 of 6 months (a, 3), control of 20 months (c, 1$)$, and Tg2576 of 20 months (c, 3).. "Before $\mathrm{KCl}$ " is the first image of the time-lapse sequence. "After $\mathrm{KCl}$ " is the last image of the timelapse sequence. "Difference image" is the image obtained by subtracting the last image to the first of the time-lapse sequence. $(\mathbf{a}, 2 ; \mathbf{a}, 4 ; \mathbf{c}, 2$; and $\mathbf{c}, 4)$ are the corresponding fluorescence intensity color map (Jet Palette) images. Brighter spots correspond to functional presynaptic terminals. b Average percentage of fluorescence loss for Control and $\operatorname{Tg} 2576$ at 6 months or $\mathbf{d}$ at 20 months. Data are derived from five independent experiments. Mean \pm s.e.m. $n=5$ experiments, *** $p<0.001$ vs. control, $t$ test. In each experiment three coverslips for each experimental group were analyzed. Scale bar $=5 \mu \mathrm{m}$

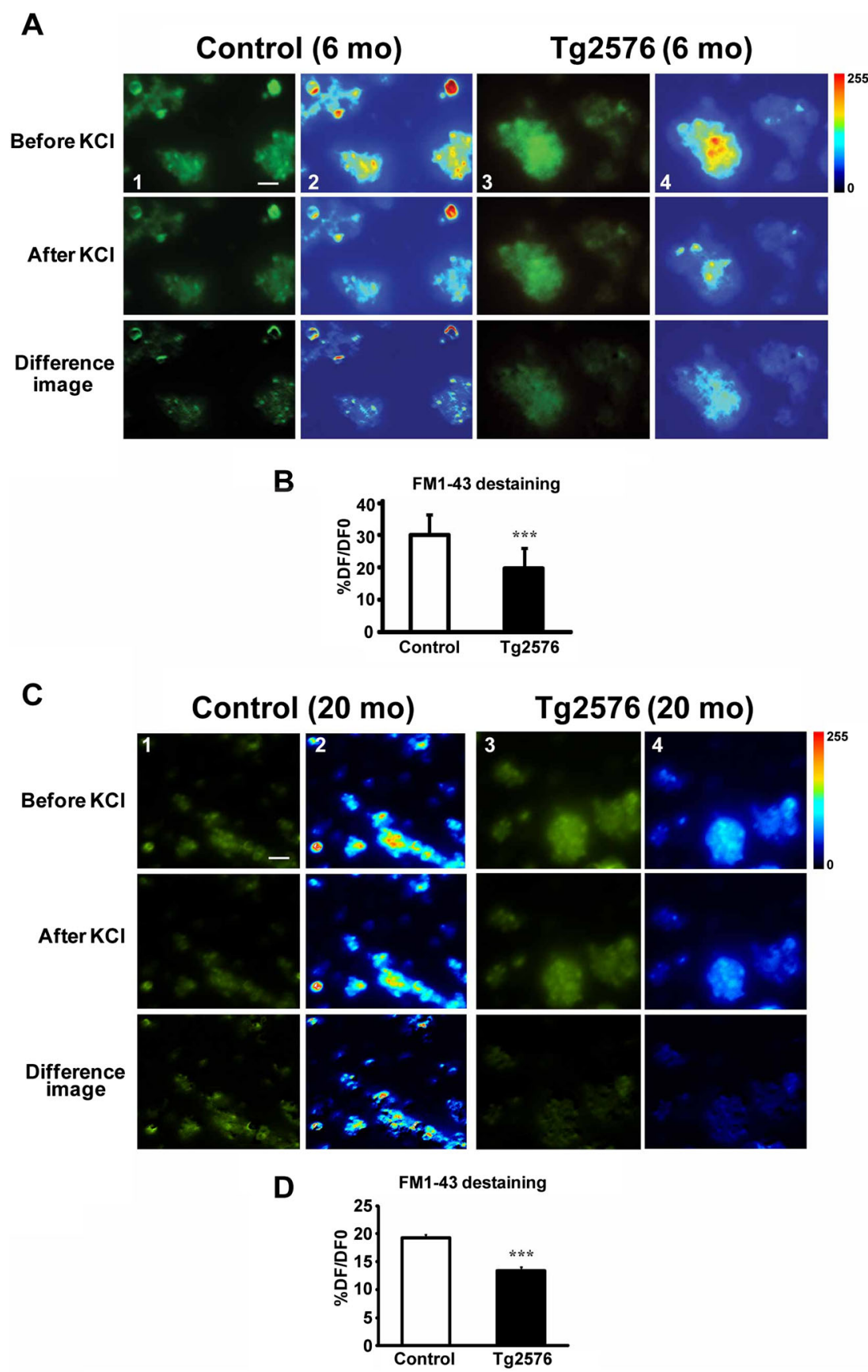

sufficient to recruit all the possible SUMO conjugations, while TU-1 alone reaches already the SUMOylation 'plateau'.

\section{TS-1 Reduces the SUMOylation of RanGAP-1 in SH-SY5Y Cell Line}

The experiments reported so far show that TS- 1 is able to reduce general SUMOylation level in a cell line and synaptosomes. To test whether the cell permeable peptide TS- 1 is able to reduce a specific known SUMOylated protein, we decided to test its effect on the SUMO conjugated RanGAP-1. Ran GTPase-activating protein 1 was the first protein identified as SUMO target [25] and it is a homodimeric $65-\mathrm{kD}$ polypeptide which helps transport other proteins from the cytoplasm to the nucleus. It has been shown that SUMO needs to be associated with it before it can be localized at the nuclear pore.

It has been reported that $8.3 \mu \mathrm{M}$ of recombinant SENP1 was able to achieve $50 \%$ deconjugation of RanGAP1SUMO1/UBC9 complex in a SUMOylation cell free assay 


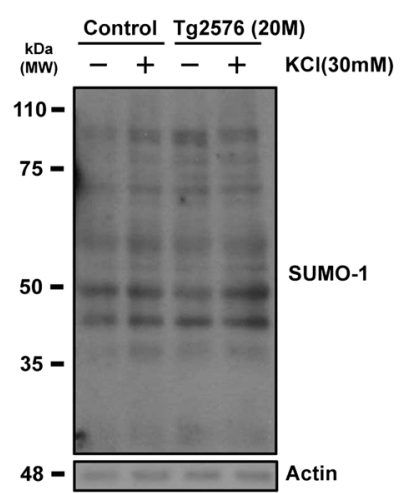

B

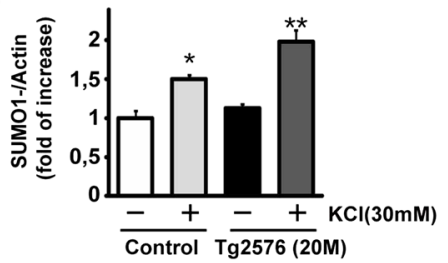

Fig. 3 Effect of $\mathrm{KCl}$ stimulus on SUMO-1 conjugation in Tg2576 at 20 months. a Representative western blot of SUMO-1 expression level. Cortical synaptosomes from $\mathrm{Tg} 2576$ mice at 20 months and age-matched controls have been stimulated with $\mathrm{KCl} 30 \mathrm{mM}$ for $90 \mathrm{~s}$, then lysed and used for western blot. $\mathrm{KCl}$ increased SUMO-1ylation in both control and Tg2576 lanes, although the induced SUMO-1ylation resulted to be more

[25]. Based on these data, SH-SY5Y cells were treated with a concentration of TS-1 higher than previous experiments, $5 \mu \mathrm{M}$ for $1.5 \mathrm{~h}$. In line with the reported data, SUMOylated RanGAP-1 was reduced by around $30 \%$ (Fig. 41: $0.75 \pm 0.04$; $* * * p<0.01$ versus control $1.09 \pm 0.05$ ) showing that the cell permeable peptide TS-1 is able to reduce the conjugation level a specific SUMOylated target protein.

\section{CPPs Modulate Synaptic Transmission}

Given the observation that the modulation of SUMO can impact on neurotransmission, the effects of CPPs have been tested on synaptic neurotransmitter release.

Interestingly, the CPPs showed an effect on FM1-43 release experiments performed on synaptosomes prepared from cortex of 6-months wild type mice. This mice age was chosen to be consistent with the data obtained from the $\mathrm{AD}$ mice model.

Cortical synaptosomes were treated with TS- 1 or TU-1, and the $30 \mathrm{mM} \mathrm{KCl-induced} \mathrm{neurotransmitter} \mathrm{release} \mathrm{was} \mathrm{assessed}$ as release of FM1-43 dye.

Our data show that CCPs were able to modulate the $\mathrm{KCl}$ evoked neurotransmitter release. In fact, $0.5 \mu \mathrm{M}$ TS-1, applied 30 min before the depolarization, incremented the neurotransmitter release of about $30 \%$ compared to $\mathrm{KCl}$ alone (Fig. $4 \mathrm{~m}$ : $1.51 \pm 0.13 ; * * *<0.001)$. This result indicates that the reduction of synaptic SUMOylation obtained by TS-1 (Fig. 4e) reflects an increase in synaptic function.
C

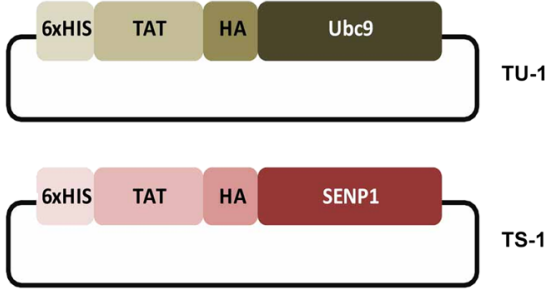

evident in AD mice. b Quantification of SUMO-1 expression level at 20 months. SUMO-1 bands signal has been normalized versus actin. Results are expressed as fold of increase vs. the SUMO-1ylation level of control. Means \pm s.e.m. $n=5$ experiments, $* p<0.05 ; * * p<0.01$ vs. control, Tukey's test. c Schematic representation of plasmids used for bacterial production of TU-1 and TS-1 recombinant fusion proteins

On the other hand, the enhancement of synaptic SUMOylation following treatment with $0.1 \mu \mathrm{M}$ TU-1 reduced the neurotransmission compared to $\mathrm{KCl}$ alone (Fig. $4 \mathrm{~m}$ : $0.76 \pm 0.18 ; * * * p<0.001)$.

\section{Modulation of SUMO Conjugation Counteracts Neurotransmission Deficit in AD Synapses}

In order to evaluate how neuronal transmission could be changed by modulation of SUMOylation in an AD mouse model, synaptosomes, prepared from cortical tissue of Tg2576 mice and their respective controls of 6 and 20 months, were subsequently treated with the CPPs. Experiments were performed measuring the FM1-43 fluorescent dye released upon $\mathrm{KCl}$ depolarization.

At 6 months, $\mathrm{Tg} 2576$ mice showed a reduced synaptic release of neurotransmitters compared to their age-matched controls (Fig. 5a: $0.62 \pm 0.08$; ***p<0.001). This result is in accordance with the augmented synaptic SUMOylation in $\mathrm{Tg} 2576$ mice at 6 months, as shown in Fig. 1a. Application of TS-1 on Tg2576 synaptosomes induced, under $\mathrm{KCl}$ stimulus, an increase in NT release which resulted to be similar to the one measured for the control (Fig. 5a: $0.90 \pm 0.06$ ). On the other hand, TU-1, reduced the release of FM1-43 respect to control, although, notably, the reduction was also significatively worsened respect to Tg2576 mice (Fig. 5a:

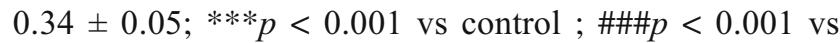
Tg2576). 

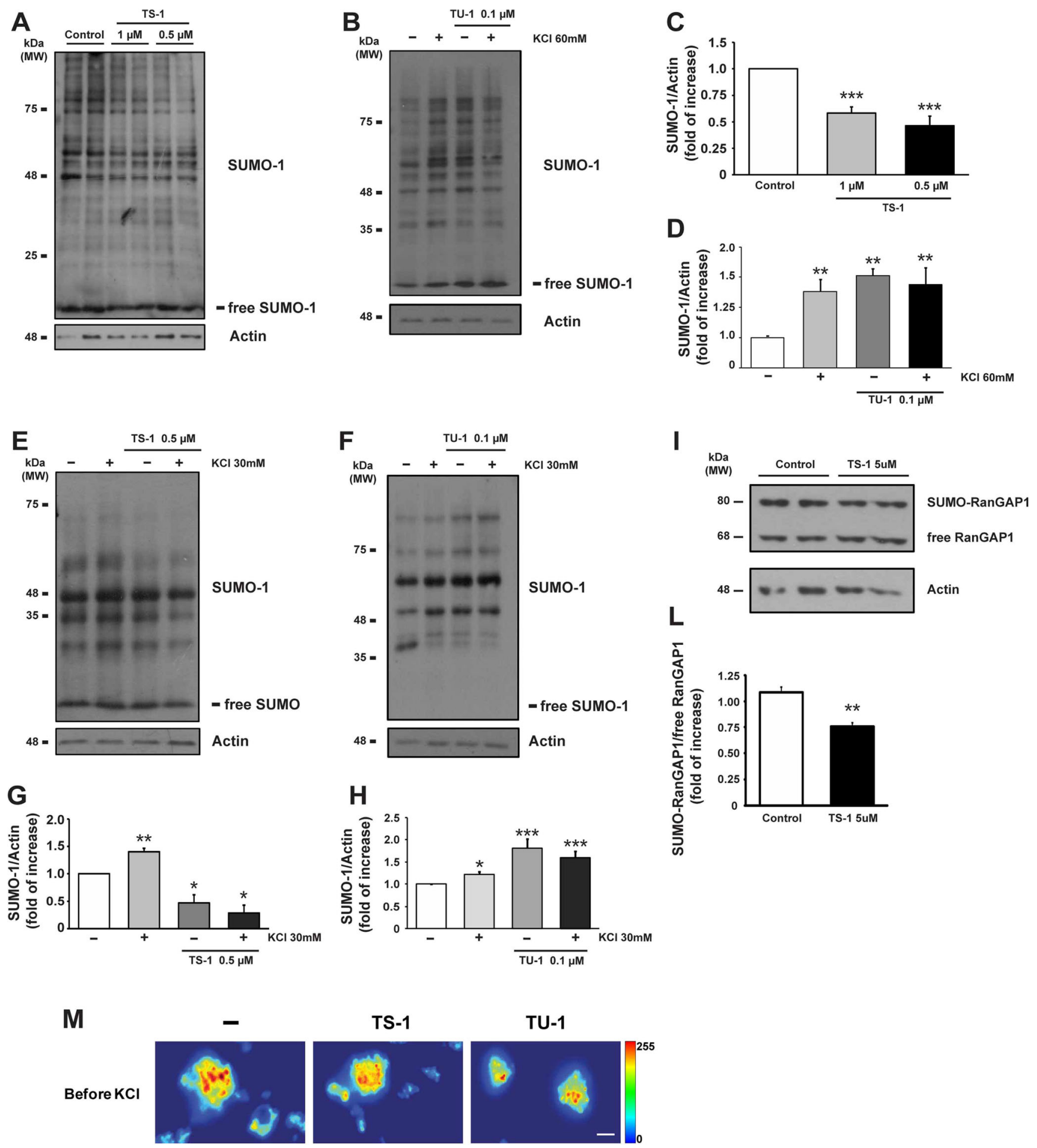

TS-1

TU-1
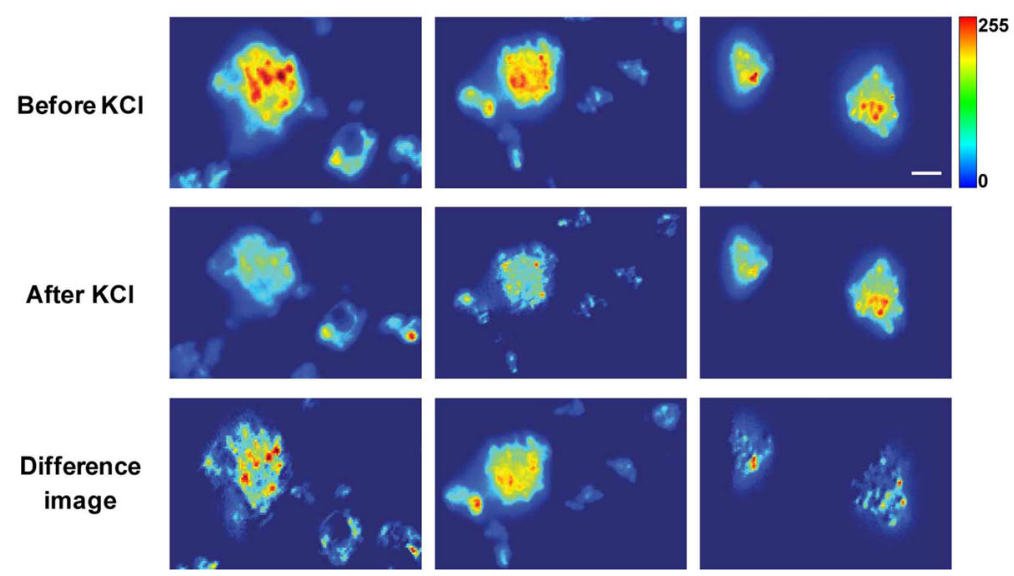

FM1-43 destaining

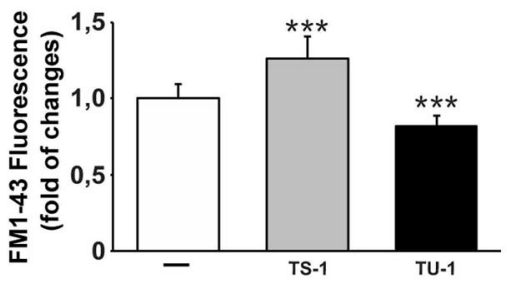


Fig. 4 CPPs treatment effect SUMO-1ylation on synaptosomes analyzed by western blot and exocytotis experiments. a-b Western blot of SUMO1 expression level in SH-SY5Y cell line pre-treated with TS-1 $(\mathbf{a}-\mathbf{c})$ or TU-1 (b-d). TS-1 was used at 1 and $0.5 \mu \mathrm{M}$ in basal conditions $1.5 \mathrm{~h}$ before harvesting the cells, while TU-1 was used at $0,1 \mu \mathrm{M}$ in both basal and $\mathrm{KCl}$ stimulated conditions $1.5 \mathrm{~h}$ before the stimulus. SUMO- 1 bands signal has been normalized versus actin. Results are expressed as fold of increase vs. the SUMO-1ylation level of control. Means \pm s.e.m. $n=5$ experiments, ${ }^{* *} p<0.01 ; * * * p<0.001$ vs. first lane, Tukey's test. (e-g) Western blot of SUMO-1 expression level of cortical synaptosomes at 6 months in several conditions. TS- 1 was applied 30 min before the $30 \mathrm{mM} \mathrm{KCl}$ stimulus. $\mathrm{KCl}$ increased basal level of SUMO-1ylation while TS-1 reduced SUMO-1ylation and prevented $\mathrm{KCl}$-induced protein conjugation. SUMO-1 bands signal has been normalized versus actin. Results are expressed as fold of increase vs. the SUMO-1ylation level of controls. Means \pm s.e.m. $n=5$ experiments, ${ }^{*} p<0.05 ; * * p<0.01$ vs. first lane with no $\mathrm{KCl}$ and no TS-1, Tukey's test. (f-h) Western blot of SUMO-1 expression level of cortical synaptosomes from wild type mice at 6 months in several conditions. TU- 1 was applied 30 min before the $30 \mathrm{mM} \mathrm{KCl}$ stimulus. $\mathrm{KCl}$ increased basal level of SUMO-1ylation while TU-1 augmented SUMO-1ylation alone. $\mathrm{KCl}$ was able to increase SUMO-1ylation in presence of TU-1 but the effect was not synergic. SUMO-1 bands signal has been normalized versus actin. Results are expressed as fold of increase vs. the SUMO-1ylation level of controls. Means \pm s.e.m. $n=5$ experiments, ${ }^{*} p<0.05 ; * * * p<0.001$ vs. first lane with no $\mathrm{KCl}$ and no TS-1, Tukey's test. (i-I) Representative western blot of SUMO-RanGAP1 deconjugation evoked by TS-1 in SH-SY5Y cultured cells. TS- 1 was applied $5 \mu \mathrm{M}$ for $1.5 \mathrm{~h}$. The peptide was able to reduce the SUMO-RanGAP1 complex immunoreactivity while the free RanGAP1 amount was increased. Means \pm s.e.m. $n=3$ experiments run in duplicates, $* * p<0.01$ vs Control. (m) Synaptogreen (FM 1-43) images of stained cortical synaptosomes stimulated with $\mathrm{KCl}$ and recorded for $30 \mathrm{~min}$ : control of 6 months (-), TS-1 pre-treatment (TS1), and TU-1 pre-treatment (TU-1). Fluorescence intensity color map (Jet Palette) images are here reported. "Before $\mathrm{KCl}$ " is the first image of the time-lapse sequence. "After $\mathrm{KCl}$ " is the last image of the time-lapse sequence. "Difference image" is the image obtained by subtracting the last image to the first of the time-lapse sequence. Quantification of FM143 fluorescence changes is shown by histograms. Mean \pm s.e.m. $n=3$ experiments, ${ }^{* *} p<0,001$ vs. control, Tukey's test. Scale bar $=5 \mu \mathrm{m}$

The CPPs activity was also tested on older Tg2576 synaptosomes, since we reported that $\mathrm{Tg} 2576$ at 20 months showed a deficit in NT release (Fig. 2b).

Although less intense than 6-months AD synapses, Tg2576 synaptosomes from 20 months showed a reduction in neurotransmitter release with respect to controls (Fig. 5b: $0.80 \pm 0.14 ; * * * p<0.001)$. Again, treatment with TS-1 prevented the synaptic deficit and the release was comparable to control (Fig. 5 b: $0.98 \pm 0.16$ ). TU-1, conversely, was not able to worsen synaptic transmission in $\mathrm{Tg} 2576$ synaptosomes, probably because the SUMOylation machinery was already at the 'plateau' of its function and could not be boosted further (Fig. 5b: $0.73 \pm 0.16 ; * * *<0.001$ ).

\section{SUMO Conjugation Is Dysregulated in Autopsy Specimens of Human Cortex with AD}

In this paper, we have shown that SUMO conjugation is significantly dysregulated in an Alzheimer's mouse model and that a fine-tuned deSUMOylation could be a potential therapeutic target. Post mortem tissue samples obtained from the frontal cortex of three demented $\mathrm{AD}$ patients along with three non-demented controls (Table S1) have been analyzed in western blot experiments to assay any SUMOylation differences. Patients were classified as 'low Braak stage' (no clinical dementia features) or 'high Braak stage' (affected by dementia) based on Braak classification [26].

This can represent a preliminary experiment on the potential translatability of our results to human Alzheimer's disease. In all frontal cortex lysates, the immunoreactivity of SUMOylation was more intense in $\mathrm{AD}$ samples than in non-AD controls (Fig. 6a: Control $=3.06 \pm 0.38$ vs $\mathrm{AD}=4.70 \pm 0.60$; $* * p<0.05)$. Moreover, synaptic fraction from the same human specimen showed an increased SUMOylation in the AD samples (Fig. 6b: Control $=2.56 \pm 0.12$ vs $\mathrm{AD}=4.87 \pm 0.28 ; * *<<0.05$ ).

The enzymes responsible for the SUMOylation machinery, namely, Ubc9 and SENP1, have been also measured in human cortical synaptosomes but no significant difference in their expression was found (Fig. $6 \mathrm{c}-\mathrm{d}$ ), indicating that the SUMOylation increase is possibly not dependent upon the overexpression of the SUMO machinery, but rather reflects a more active SUMOylation pathway.

The quality of the human synaptosomes preparation has been assessed by comparative western blot analysis with human total lysate. Several synaptic and non-synaptic protein antibodies have been used for the membrane probing. The results show that human synaptosomes do not show the presence of nuclear proteins like RanGAP-1 or the neuron-specific nuclear protein NeuN (Fig. S1a-b) while they are instead present in the human total lysate. As expected, synaptic proteins like PSD-95, Syntaxin-1a, and Synaptotagmin-1 are instead enriched in the synaptosome preparation respect to total lysates (Fig. S1c-e).

\section{Discussion}

In our study, we have demonstrated that synaptic SUMOylation is altered in the Tg2576 mouse model of AD at 6 and 20 months of age

Our results are consistent with previous reports in which SUMOylation was demonstrated to interfere with synaptic function [15]. Indeed, synaptic impairment has been described as the first neuronal deficit at the onset of the AD. The severity of synaptic loss is correlated with memory impairment and dementia during $\mathrm{AD}$ progression. Amyloid $\beta$ protein $(\mathrm{A} \beta)$ is strongly implicated in the pathogenesis of $A D$, being $A \beta$ oligomers heavily synaptotoxic [27]. The early synaptic dysfunction has been also correlated with caspase-3 activity in hippocampal areas wherein the enhancement of its activity seems to lead to the onset of memory decline in 3-months old Tg2576 mice [7]. A $\beta$ oligomers interfere at synaptic level 


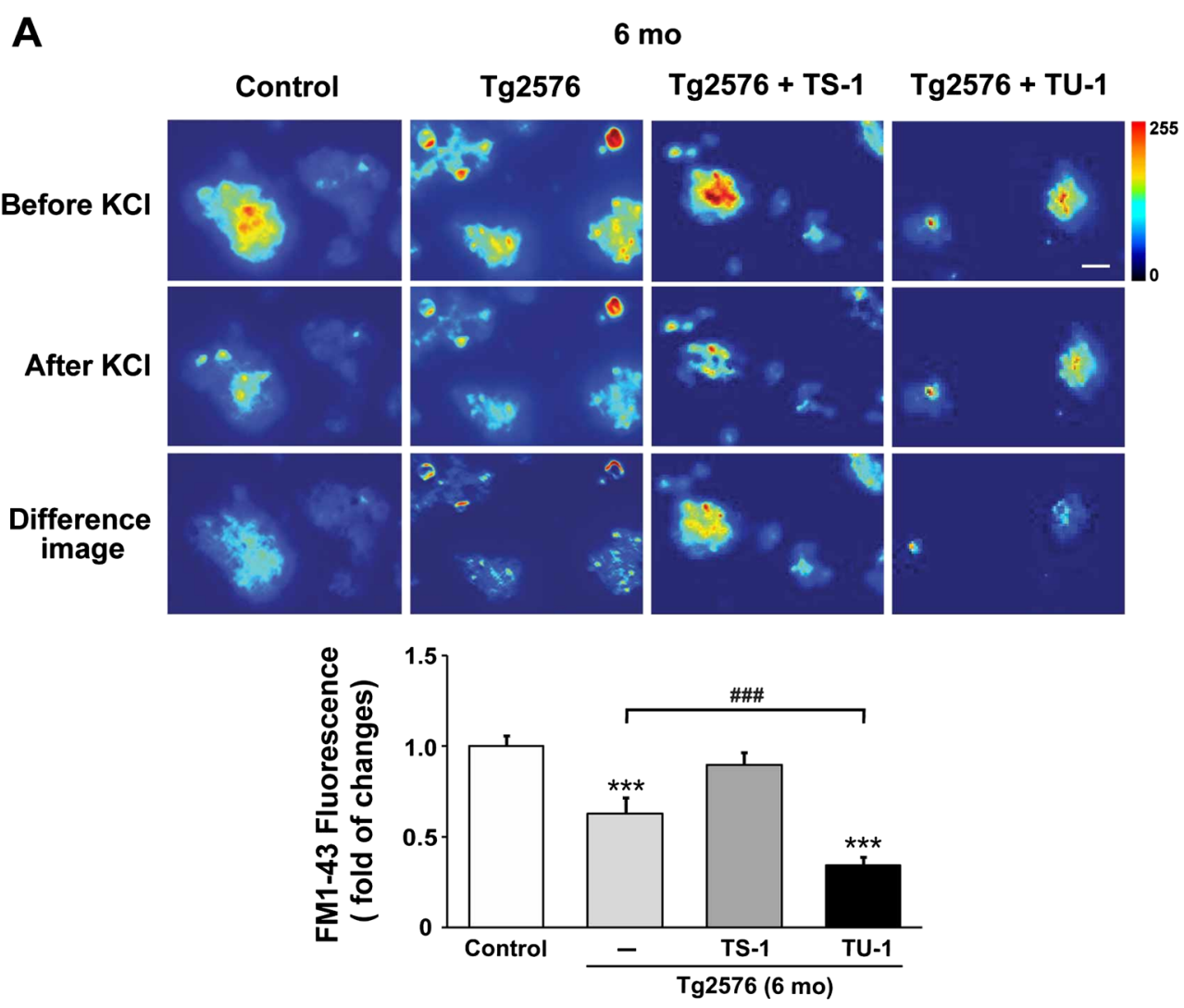

B

20 mo
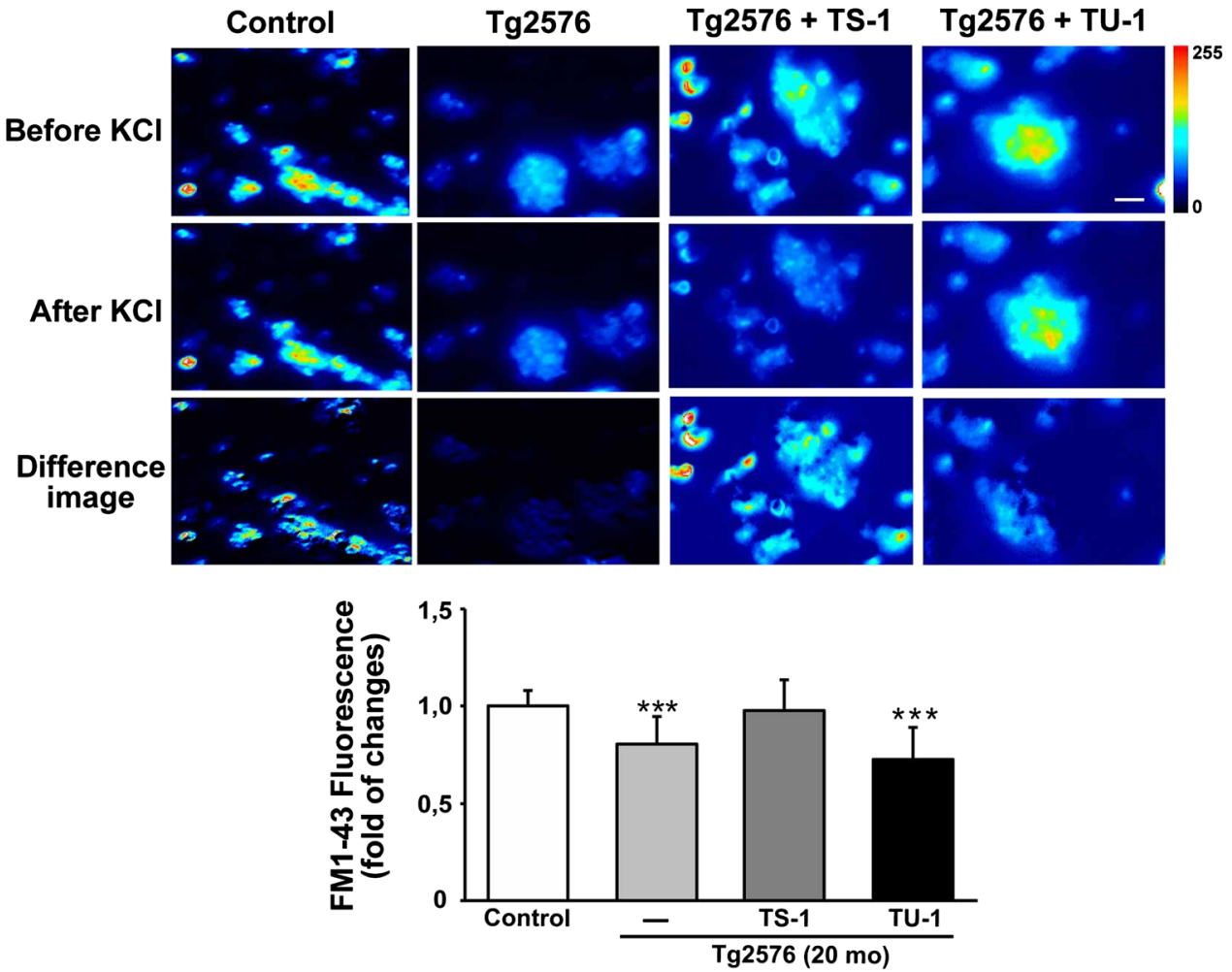
Fig. 5 CPPs pre-treatment effect on synaptosomes of Tg2576 at 6 and 20 months during exocytotis experiments. Synaptogreen (FM 1-43) images of stained cortical synaptosomes of Tg2576 at 6 and 20 months mice stimulated after $90 \mathrm{~s}$ with $\mathrm{KCl}$ and recorded for $30 \mathrm{~min}$ : a control of 6 months (Control), Tg2576 of 6 months (Tg2576), Tg2576 of 6 months + TS-1 pre-treatment $(\mathrm{Tg} 2576+\mathrm{TS}-1)$, and $\mathrm{Tg} 2576$ of 6 months + TU-1 pre-treatment $(\mathrm{Tg} 2576+\mathrm{TU}-1)$. b Control of 20 months (Control), Tg2576 of 20 months (Tg2576), Tg2576 of 20 months + TS-1 pre-treatment $(\mathrm{Tg} 2576+\mathrm{TS}-1)$, and $\mathrm{Tg} 2576$ of 20 months + TU-1 pre-treatment $(\mathrm{Tg} 2576+\mathrm{TU}-1)$. Fluorescence intensity color map (Jet Palette) images are here reported. "Before $\mathrm{KCl}$ " is the first image of the time-lapse sequence. "After $\mathrm{KCl}$ " is the last image of the time-lapse sequence. "Difference image" is the image obtained by subtracting the last image to the first of the time-lapse sequence. Quantification of FM1-43 fluorescence changes is shown by histograms. Mean \pm s.e.m. $n=4$ experiments, $* * * p<0.001$ vs. control, Tukey's test. Scale bar $=5 \mu \mathrm{m}$

with synaptic plasticity events, favoring over time the degeneration of synapses [28, 29]. Synaptic neurotransmission in AD is highly compromised; acetylcholine (ACh), for instance, is reduced in $\mathrm{AD}$ patients whereas a glutamate-driven overactivation of N-methyl-d-aspartate (NMDA) receptors contributes to excitotoxicity and synaptic loss [30]. In Tg2576 mice, neither reduction in post-synaptic AMPA nor KA receptor was measured, therefore it has been postulated that the synaptic transmission deficit could be supported by a reduced presynaptic release [31].

SUMO-1ylation has been previously correlated with neurotransmission. The increase of SUMOylation at the synapses, obtained by entrapping recombinant SUMO-1 in synaptosomes, induces a reduction in glutamate release. On the other hand, by reducing SUMOylation with recombinant SENP1, glutamate release is increased [17].

Previous data showed that SUMO-1ylation levels in $\operatorname{Tg} 2576$ mice are augmented at 3 and 6 months, which correspond to an early stage of the pathology. Conversely, the level of SUMO-1ylation was not different from controls in 17 months old transgenic mice [19]. SUMO-2/3ylation did not show meaningful differences between $\mathrm{Tg} 2576$ mice and controls up to 6 months, although it was later reduced at 17 months.

According to these data, synaptosomes from cortical tissue of 6-mo Tg2576 mice presented an increased SUMO1ylation, while at 20 months, we did not found any difference compared to control.

Interestingly, the augmented mRNA expression level that was found in total tissue lysate [19], has been confirmed by confocal images we performed at 6 months.
Fig. 6 SUMO-1ylation level in human $\mathrm{AD}$ frontal cortex lysate and synaptosomes. Western blot of SUMO-1 expression level in frontal cortex from human lysates (a) and frontal cortex synaptosomes (b). Control lanes are referred to as 'low Braak stage' human specimens, while $\mathrm{AD}$ patients are classified as 'high Braak stage' samples and characterized by clinically verified dementia. Histograms report the quantification of SUMO-1ylation fold of increase. SUMO-1 bands signal has been normalized versus actin. Results are expressed as fold of increase vs. control. Means \pm s.e.m. $n=5$ experiments, ${ }^{*} p<0.05, t$ test. Western blot of Ubc9, SENP1 expression level in frontal cortex human synaptosomes (c) and A $\beta$ PP expression level in frontal cortex human synaptosomes (d)
A
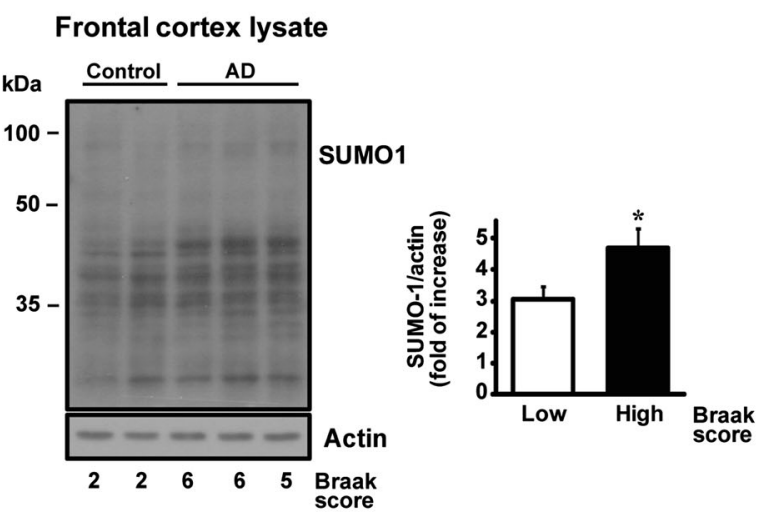

B

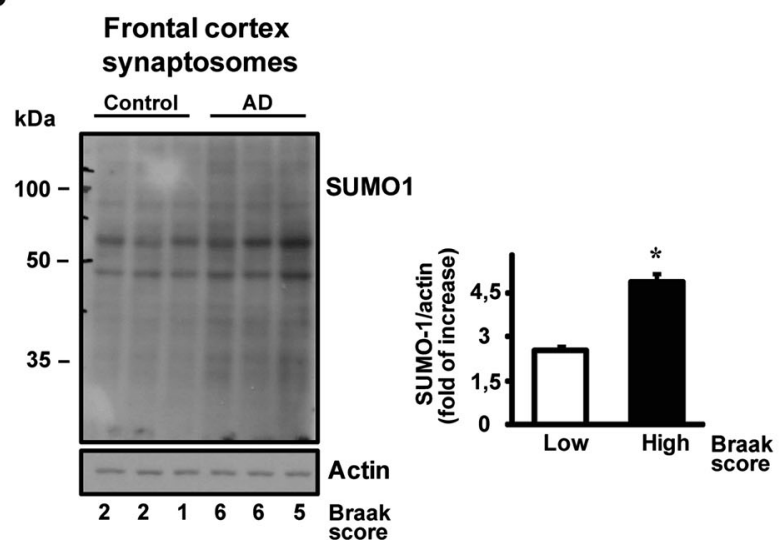

C

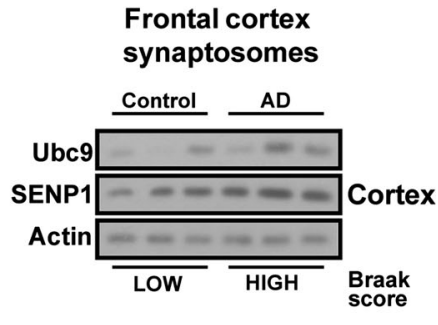

D

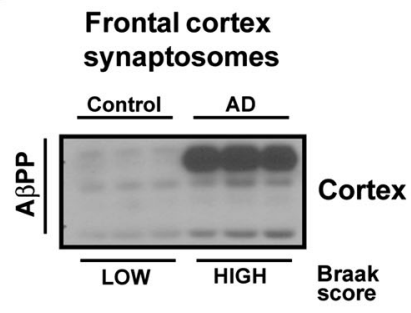


Synaptic SUMO-2ylation resulted to regulate synaptic plasticity and cognition in both normal and Alzheimer's disease conditions. In fact, increasing SUMO-2ylation via transduction of its conjugating enzyme, Ubc 9 , rescued $A \beta$-induced deficits in LTP and hippocampal-dependent learning and memory [15]. In these reports, authors did not observe any alteration involving the SUMO-1 paralog in lysate from $\mathrm{AD}$ mice at 7-8 and 13-14 months. Their result was in line with our previous observation showing that after 6-monthsSUMO1ylation of AD mice is not different from control [19]. Since our preliminary data showed an increased synaptic SUMO1ylation, we then evaluated the neurotransmitter release activity in Tg2576 mice of 6 and 20 months. $\mathrm{KCl}$-evoked vesicles release was induced and, in addition, it was found that the augmented SUMO-1ylation at 6 months corresponded to a decreased synaptic activity. $\mathrm{KCl}$ is known to induce an increase of basal SUMO conjugation at the synapse [15, 17]. In fact, although 20-months old Tg2576 did not show SUMOylation level differences, the released fluorescent dye was reduced in Tg2576 mice, and the SUMO-1ylation level under stimulus was augmented with respect to control mice. This observation fostered the hypothesis that SUMO-1 conjugation level is more susceptible in the synapses of 20-months old Tg2576 compared to their respective controls. The role of SUMOylation as modulator of synaptic transmission that we here emphasized is also supported by a recent work in which SUMO-1 overexpressing mice showed an impaired synaptic function in an age-dependent manner. Probably, as we hypothesized, a continued elevated SUMOylation throughout mice life is able to cause the molecular changes that leads lately into a reduction of synaptic functions [18].

In order to study the role of SUMOylation on synaptic transmission, we then manipulated SUMOylation levels. Ginkgolic acid or anacardic acid are the only SUMOylation inhibitors available yet, however, it is not clear how specific they are [32]. Notably, both these natural compounds are currently investigated in clinical trials related to Alzheimer's patients, although only minimal benefits have been observed so far and further evidences will be required in the future [33].

Consequently, we produced two cell permeable peptides able to selectively increase (TU-1) or decrease (TS-1) protein SUMOylation. These two proteins carry an effector portion which is represented respectively by the SUMOylation enzyme Ubc9 and the functional core of SENP1. Plasmids overexpressing both enzymes have been previously used to modulate SUMOylation in cell lines [24].

Tat-based permeable peptides are extensively used in basic research in order to modulate biological processes [24, 34, 35].

TS-1 was able to reduce protein SUMO-1ylation in control synaptosomes in both basal and $\mathrm{KCl}$ stimulated conditions. As result, and in accordance with the previous evidences [17], the reduction in protein SUMOylation increased synaptic transmission measured from cortical synaptosomes.
TU-1 was previously used by other authors, even though an activity of this peptide on SUMO-1ylation was not observed [19]. In our hands, TU-1 increased basal SUMO1ylation but not $\mathrm{KCl}$-stimulated SUMO-1ylation, showing that SUMOylation system had probably already reached a 'plateau' phase. In fact, $\mathrm{KCl}$-evoked NT release was reduced by TU-1, although the difference was not large. Even if our results seem to be in contrast with previously described data, we here used synaptosomes that indeed represent a very different system model compared to brain slices [19].

SUMO modulation has been therefore tested on Tg2576 synaptosomes to understand whether the two cell permeable peptides could be able to modify and possibly rescue the synaptic deficit.

Intriguingly, TS-1 proved to abolish the reduction of $\mathrm{KCl}-$ evoked neurotransmitter release, as shown by synaptosomes from both 6 and 20 month old Tg2576 with respect to wildtype controls. On the contrary, TU-1 further worsened synaptic transmission.

Notably, for the first time, we here show that SUMO1ylation is increased in both brain lysate and synaptosomes from the cortex of $\mathrm{AD}$ patients. This observation holds translational implication, since the cognitive and mnemonic deficits reported in $\mathrm{AD}$ patients could be somehow correlated with SUMOylation alterations at the synapse. Our results using CPPs strongly encourage us to postulate that a potential drug able to modulate synaptic SUMOylation could help to restore synaptic dysfunction in AD. Certainly, further studies are required to elucidate the SUMO targets at the synaptic level. This is a fundamental task in order to design novel and specific SUMO-based drugs able to re-establish at an early stage normal synaptic communication in $\mathrm{AD}$ and related dementias.

\section{Materials and Methods}

\section{Animals and Brain Tissue Preparation}

Tg2576 mice at 6 and 20 months of age were killed by decapitation in accordance with the guidelines established by the European Communities Council (Directive 2010/63/EU of 22 September 2010) and accepted by the Italian Ministry of Health and approved by the Ethical Committee on animal experiments of EBRI "Rita Levi-Montalcini" Foundation (Rome, Italy). Brains were rapidly dissected on ice to preserve brain integrity during sample collection. All the procedures necessary to prepare the brain fractions were performed at $0-4{ }^{\circ} \mathrm{C}$.

The AD mouse model Tg2576, carrying the double Swedish mutation (K670 N and M671 L) [36], recapitulates some of the human pathology features, like overproduction of $\mathrm{A} \beta_{40}$ and $\mathrm{A} \beta_{42}$ and $\mathrm{A} \beta$ deposition in the frontal, temporal, and entorhinal cortices, hippocampus, presubiculum, and cerebellum at about 11-13 months of age [37]. 


\section{Human Brain Samples}

Human brains were obtained by autopsy with a post mortem delay of $12.8 \pm 13.2 \mathrm{~h}(2-32 \mathrm{~h})$. The series included 6 subjects (mean age: $87.3 \pm 4.7$ years), 3 with $\mathrm{AD}$ ( 2 with Braak stages 6 and one with Braak stage 5; mean age: $91.0 \pm 3$.6 years) and 3 without Alzheimer-type pathology (mean age: $83.6 \pm 1.5$ years) on neuropathological examination. For the present study, frozen tissues from the frontal cortex were used (see table in supplementary).

\section{Synaptosomal Preparation}

Purified synaptosomes were prepared as previously described [38-40]. Briefly, the tissue, murine or human, was homogenized in 10 volumes of $0.32 \mathrm{M}$ sucrose, buffered to $\mathrm{pH} 7.4$ with Tris-(hydroxymethyl)-amino methane [Tris, final concentration (f.c.) $0.01 \mathrm{M}$ ] using a glass/Teflon tissue grinder (clearance $0.25 \mathrm{~mm}$ ); the homogenate was centrifuged at $1000 \times g$ for $5 \mathrm{~min}$ to remove nuclei and debris, and the supernatant was gently stratified on a discontinuous Percoll (Sigma Aldrich, Italy) gradient $(2,6,10$ and $20 \% \mathrm{v} / \mathrm{v}$ in Tris-buffered sucrose) and centrifuged at $33,500 \times \mathrm{g}$ for $5 \mathrm{~min}$. The layer between 10 and $20 \%$ Percoll (synaptosomal fraction) was collected and washed by centrifugation. The synaptosomal pellets were resuspended in a physiological solution (Saline Buffer) with the following composition (mM): $\mathrm{NaCl}, 140$; $\mathrm{KCl}, 3 ; \mathrm{MgSO} 4,1.2 ; \mathrm{CaCl} 2,1.2 ; \mathrm{NaH} 2 \mathrm{PO} 4,1.2 ; \mathrm{NaHCO} 3$, 5; HEPES, 10; glucose, 10; and $\mathrm{pH}$ 7.2-7.4.

\section{SH-SY5Y Cell Culture}

SH-SY5Y human neuroblastoma cells have been seeded into 6 -well plates at density of $4 \times 10^{5} 1$ day before treatments and were maintained in complete medium with the following composition: DMEM (LONZA Group LTD, Switzerland) $4.5 \mathrm{~g} / \mathrm{L}$ glucose, $10 \%$ fetal bovine serum (LONZA Group LTD, Switzerland), $1 \%$ L-glutamine (Invitrogen - GIBCO) $200 \mathrm{mM}, 1 \%$ antibiotics penicillin/streptomycin (Invitrogen - GIBCO), 1 \% MEM non-essential amino acids solution $100 \times($ SIGMA).

Cells were incubated at $37{ }^{\circ} \mathrm{C}$ under $5 \% \mathrm{CO}_{2}$.

\section{Measurements of Synaptic Vesicle Exocytosis}

Synaptogreen (SIGMA-ALDRICH, Italy), also known as FM1-43, was used to measure synaptic release as previously described $[20,41,42]$. Synaptosomes $(0.3 \mathrm{mg} / \mathrm{mL})$ were incubated in $2 \mathrm{ml}$ of Saline Buffer for $2 \mathrm{~min}$ at $37{ }^{\circ} \mathrm{C}$ in mild agitation. Synaptogreen $(50 \mu \mathrm{M})$ was added and after $3 \mathrm{mi}-$ nutes, stimulation with $\mathrm{KCl}(30 \mathrm{mM})$ was applied to load the fluorescent dye. After $90 \mathrm{~s}$ stimulation, synaptosomes were washed twice to remove non-internalized Synaptogreen in
Saline Buffer containing $1 \mathrm{mg} / \mathrm{mL}$ BSA. Synaptosomes ( $200 \mu \mathrm{g}$ of proteins) were then resuspended in $2 \mathrm{~mL}$ and seeded on Poly-L-Lysine $(50 \mu \mathrm{g} / \mathrm{ml})$ treated coverslips. After 40 min, two Saline Buffer washes followed to remove not seeded synaptosomes, and coverslips were placed on a time-lapse system mounted on an inverted fluorescence microscope (TiE; Nikon, Japan), equipped with an incubation chamber (Okolab), a cooled CCD camera (Clara; Andor), Perfect Focus System to avoid z-axis focus fluctuations and a Niss Elements imaging software (Nikon). Video recordings were performed with a $40 \times$ oil objective (N. A 1.4) for $6 \mathrm{~min}$ $(30 \mathrm{mM} \mathrm{KCl})$ by taking 14 bit images every $2 \mathrm{~s}$. at room temperature $\left(25^{\circ} \mathrm{C}\right)$. Fluorescence was recorded and video images were analyzed by using the Spot module of the ImarisSuite 7.6® software (Bitplane A.G., Zurich, Switzerland) by selecting a $1.5 \mu \mathrm{m}$ radius diameter of fluorescent puncta. Fluorescence intensity was measured in 15-20 randomly selected synaptosomes within the microscopic field. The criteria for fluorescence puncta inclusion in the data analysis were the spherical shape ranging between $0.5-1.5 \mu \mathrm{m}$ and the stimulation-dependent destaining response. Destaining time courses were generated by normalization of each fluorescence spot trace. Quantification of FM1-43 responses was accomplished by calculating the average percentage of fluorescence loss. A group of control experiments was run to test the specificity of Synaptogreen loading and destaining. $\mathrm{Ca}^{2+}$ free medium drastically reduced the dye loading and inhibited the stimulus-induced dye release. The Synaptogreen photobleaching rate was also measured in the absence of the stimulus and compared to the stimulated Synaptogreen destaining traces for each experimental group.

\section{Cell Permeable Peptides Preparation}

Tat-Ubc9 (TU-1) and Tat-SENP1 (TS-1) constructs were made by subcloning human Ubc9 or human SENP1 respectively from Flag-Ubc9 and pEYFP-SENP1 [24] into a TatHA-6xHis vector (Addgene, USA). SENP1 sequence is referred to the full catalytic domain of the enzyme corresponding to 351-644 amino acid sequence. Protein expression in E. coli and purification via the $6 \mathrm{xHis}$ tag was performed according to standard procedures previously described [43].

\section{Stimulation of Synaptosomes or SH-SY5Y Cell Line in Biochemical Experiments}

Stimulation of synaptosomes was performed in batch as previously reported [20]. Re-suspended synaptosomes were divided into $0.2 \mathrm{ml}$ aliquots and gently agitated at $37^{\circ} \mathrm{C}$ until stimulation. Following $10 \mathrm{~min}$ mild agitation at $37^{\circ} \mathrm{C}$, TS- 1 $(0.5 \mu \mathrm{M})$ or TU-1 $(0.1 \mu \mathrm{M})$ were added as indicated and left for $20 \mathrm{~min}$ at $37^{\circ} \mathrm{C}$ before stimulus and until the end of the experiments. $\mathrm{KCl}$ stimulus was added for $90 \mathrm{~s}$, then $0.2 \mathrm{ml}$ of 
warm medium containing inhibitors, where needed, was added until the end of the experiment.

The experiments were terminated by adding $0.6 \mathrm{ml}$ of cold Saline Buffer and immediate centrifugation at $12,000 \times g$ to pellet the synaptosomes.

The final pellets were lysed in $\sim 100 \mu$ of Lysis Buffer solution (LB) made up of $1 \%$ Triton X-100 (Serva, Germany), complete protease inhibitor cocktail solution (Serva, Germany), phosphatase inhibitor cocktail solution (Serva, Germany), and the following components (mM): TRIS acetate, 20; sucrose, 0.27; EDTA, 1; EGTA, 1; Na Orthovanadate, $1 ; \mathrm{NaF}, 50 ; \mathrm{Na}$ Pyrophosphate, $5 ; \mathrm{Na} \beta$-glycerophosphate, 10; and DTT, 1.

SH-SY5Y cells were treated with TS-1 (different concentrations) or TU-1 $(0,1 \mu \mathrm{M}) 1 \mathrm{~h}$ and $30 \mathrm{~min}$ before the $\mathrm{KCl}$ $(60 \mathrm{mM})$ stimulus. $\mathrm{KCl}$ has been administered for $90 \mathrm{~s}$ and after two Phosphate buffer (PBS) washes, cells have been lysed in $\sim 150 \mu$ of LB.

Protein concentration was then determined by Bradford assay and the samples were directly analyzed by immunoblotting and following resuspension in Laemmli buffer.

\section{Immunofluorescence}

Immediately after preparation, synaptosomes were seeded on coverslip coated with Poly-L-Lysine $(50 \mu \mathrm{g} / \mathrm{ml})$, washed twice with PBS and fixed in $4 \%(w / v)$ freshly prepared paraformaldehyde in PBS for $10 \mathrm{~min}$ at room temperature. After four washes, synaptosomes were permeabilized with $0.05 \%$ $(v / v)$ Triton X-100/PBS for $5 \mathrm{~min}$, washed and then incubated overnight in a mix solution of the following primary antibodies: rabbit anti-SUMO-1 (Cell Signaling, USA) diluted 1:200 in $0.05 \%(\mathrm{v} / \mathrm{v})$ Triton X-100/PBS at $4{ }^{\circ} \mathrm{C}$. Unbound antibody was removed by four $10 \mathrm{~min}$ long washes with PBS at room temperature. Bound antibody was detected by incubation with donkey anti-rabbit Alexa 488 (1:500) conjugated secondary antibody (Invitrogen, USA) at $4{ }^{\circ} \mathrm{C}$ for $2 \mathrm{~h}$. After three washes with PBS at room temperature, coverslips were mounted on slides with Hydromount ${ }^{\mathrm{TM}}$ mounting gel. Controls were performed by omitting the primary antibodies.

\section{Western Blot}

Equal amount of proteins ( $15 \mu \mathrm{g}$ for each condition) were resolved by $10 \%$ SDS-polyacrylamide gels and blotted onto PVDF membrane (Serva, Germany). The resulting blot was blocked for $1 \mathrm{~h}$ at room temperature using Tris-buffered saline-Tween (t-TBS) (M) Tris, 0.02; $\mathrm{NaCl}, 0.15$; Tween 20, $0.1 \%$ ) containing $5 \%$ skimmed milk. The membranes were then incubated overnight at $4{ }^{\circ} \mathrm{C}$ with specific antibodies: rabbit anti-SUMO-1 1:1000 (Cell Signaling, USA), rabbit anti-Ubc9 1:1000 (Santa Cruz, USA), rabbit anti-SENP1 1:500 (Thermo Scientific, USA), rabbit anti-AßPP 1:1000
(Sigma-Aldrich, USA), rabbit anti-RanGAP1 1:1000 (Abgent, USA), anti rabbit anti-Syntaxin 1a 1:1000 (Synaptic System, Germany), mouse anti-PSD95 1:1000 (Santa Cruz, USA), mouse anti-NeuN 1:1000 (Millipore, USA), mouse anti-Synaptotagmin 1 1:1000 (Santa Cruz, USA), and mouse anti-Actin 1:1000 (Sigma-Aldrich, USA).

After $50 \mathrm{~min}$ of wash in t-TBS, the blots were incubated for $1 \mathrm{~h}$ at room temperature with peroxidase-conjugated goat anti-rabbit or anti-mouse IgG secondary antibodies (UCS Diagnostic).

After 50 min washes in t-TBS, band immunoreactivity was detected by enhanced chemiluminescence (ECL; WESTAR, Cyanagen, Italy).

\section{Statistical Analysis}

In release experiments, analysis of variance was performed by ANOVA followed by Newman-Keuls multiple-comparison test. When comparing only a single treatment group, a Student's two-tailed $t$ test was used. Data were considered significant for $p<0.05$ at least. Appropriate controls with enzyme inhibitors were always run in parallel.

Statistical analysis for biochemical experiments was performed with GraphPad PRISM 4 (GraphPad Software, USA). Values shown represent the mean \pm SEM of a different number of separate experiments (as indicated in figure legends). One-way or Two-ways ANOVA followed by Tukey's test was carried out when intergroup comparisons were required.

Acknowledgments We thank Lundbeck A/S (Copenhagen, Denmark) for kindly providing $\mathrm{Tg} 2576$ mice samples and respective controls. This work has been financially supported by Lundbeck A/S grant and partially by 'Ricerca Corrente 2013-2016' from Istituto C. Mondino (Pavia, Italy).

\section{References}

1. Holtzman DM, Morris JC, Goate AM (2011) Alzheimer's disease: the challenge of the second century. Sci Transl Med 3:77-77sr1

2. Braak H, Braak E (1991) Neuropathological stageing of Alzheimerrelated changes. Acta Neuropathol 82(4):239-259

3. Serrano-Pozo A, Frosch MP, Masliah E, Hyman BT (2011) Neuropathological alterations in Alzheimer disease. Cold Spring Harb Perspect Med 1(1):a006189

4. Overk CR, Masliah E (2014) Pathogenesis of synaptic degeneration in Alzheimer's disease and Lewy body disease. Biochem Pharmacol 88(4):508-516

5. Sheng M, Sabatini BL, Südhof TC (2012) Synapses and Alzheimer's disease. Cold Spring Harb Perspect Biol 4(5)

6. Krafft GA, Klein WL (2010) ADDLs and the signaling web that leads to Alzheimer's disease. Neuropharmacology 59(4-5):230 242

7. D'Amelio M, Cavallucci V, Middei S, Marchetti C, Pacioni S, Ferri A, Diamantini A, De Zio D et al (2011) Caspase-3 triggers early synaptic dysfunction in a mouse model of Alzheimer's disease. Nat Neurosci 14(1):69-76 
8. Louneva N, Cohen JW, Han LY, Talbot K, Wilson RS, Bennett DA, Trojanowski JQ, Arnold SE (2008) Caspase-3 is enriched in postsynaptic densities and increased in Alzheimer's disease. Am J Pathol 173(5):1488-1495

9. Wu H-Y, Hudry E, Hashimoto T, Kuchibhotla K, Rozkalne A, Fan Z, Spires-Jones T, Xie H et al (2010) Amyloid beta induces the morphological neurodegenerative triad of spine loss, dendritic simplification, and neuritic dystrophies through calcineurin activation. J Neurosci 30(7):2636-2649

10. Lee L, Sakurai M, Matsuzaki S, Arancio O, Fraser P (2013) SUMO and Alzheimer's disease. Neruomol Med 15(4):720-736

11. Sarge KD, Park-Sarge O-K (2011) SUMO and its role in human diseases. Int Rev Cell Mol Biol 288:167-183

12. Droescher M, Chaugule VK, Pichler A (2013) SUMO rules: regulatory concepts and their implication in neurologic functions. Neruomol Med 15(4):639-660

13. Feligioni M, Marcelli S, Knock E, Nadeem U, Arancio O, Fraser PE (2015) SUMO modulation of protein aggregation and degradation. AIMS Mol Sci 2(4):382-410

14. Feligioni M, Nisticò R (2013) SUMO: a (oxidative) stressed protein. Neruomol Med 15(4):707-719

15. Lee L, Dale E, Staniszewski A, Zhang H, Saeed F, Sakurai M, Fa M, Orozco I et al (2014) Regulation of synaptic plasticity and cognition by SUMO in normal physiology and Alzheimer's disease. Sci. Rep. 4:7190

16. Martin S, Nishimune A, Mellor JR, Henley JM (2007) SUMOylation regulates kainate-receptor-mediated synaptic transmission. Nature 447(7142):321-325

17. Feligioni M, Nishimune A, Henley JM (2009) Protein SUMOylation modulates calcium influx and glutamate release from presynaptic terminals. Eur J Neurosci 29(7):1348-1356

18. Matsuzaki S, Lee L, Knock E, Srikumar T, Sakurai M, Hazrati L-N, Katayama T, Staniszewski A et al (2015) SUMO1 affects synaptic function, spine density and memory. Sci. Rep. 5:10730

19. Nisticò R, Ferraina C, Marconi V, Blandini F, Negri L, Egebjerg J, Feligioni M (2014) Age-related changes of protein SUMOylation balance in the A $\beta$ PP Tg2576 mouse model of Alzheimer's disease. Front Pharmacol 5:63

20. Nisticò R, Florenzano F, Mango D, Ferraina C, Grilli M, Di Prisco S, Nobili A, Saccucci S et al (2015) Presynaptic c-Jun N-terminal Kinase 2 regulates NMDA receptor-dependent glutamate release. Sci Rep 5:9035

21. Lu H, Liu B, You S, Xue Q, Zhang F, Cheng J, Yu B (2009) The activity-dependent stimuli increase SUMO modification in SHSY5Y cells. Biochem Biophys Res Commun 390(3):872-876

22. Gong B, Cao Z, Zheng P, Vitolo OV, Liu S, Staniszewski A, Moolman D, Zhang H et al (2006) Ubiquitin hydrolase Uch-L1 rescues beta-amyloid-induced decreases in synaptic function and contextual memory. Cell 126(4):775-788

23. Gump JM, Dowdy SF (2007) TAT transduction: the molecular mechanism and therapeutic prospects. Trends Mol Med 13(10): 443-448

24. Feligioni M, Brambilla E, Camassa A, Sclip A, Arnaboldi A, Morelli F, Antoniou X, Borsello T (2011) Crosstalk between JNK and SUMO signaling pathways: deSUMOylation is protective against $\mathrm{H} 2 \mathrm{O} 2$-induced cell injury. PLoS One 6(12):e28185

25. Gareau JR, Reverter D, Lima CD (2012) Determinants of small ubiquitin-like modifier 1 (SUMO1) protein specificity, E3 ligase, and SUMO-RanGAP1 binding activities of nucleoporin RanBP2. J Biol Chem 287(7):4740-4751

26. Alafuzoff I, Arzberger T, Al-Sarraj S, Bodi I, Bogdanovic N, Braak H, Bugiani K, Del-Tredici K et al (2008) Staging of neurofibrillary pathology in Alzheimer's disease: a study of the BrainNet Europe consortium. Brain Pathol 18(4):484-496

27. Shankar GM, Walsh DM (2009) Alzheimer's disease: synaptic dysfunction and Abeta. Mol Neurodegener 4(1):48

28. Nisticò R, Pignatelli M, Piccinin S, Mercuri NB, Collingridge G (2012) Targeting synaptic dysfunction in Alzheimer's disease therapy. Mol Neurobiol 46(3):572-587

29. Nimmrich V, Ebert U (2009) Is Alzheimer's disease a result of presynaptic failure? Synaptic dysfunctions induced by oligomeric beta-amyloid. Rev Neurosci 20(1):1-12

30. Francis PT (2005) The interplay of neurotransmitters in Alzheimer's disease. CNS Spectr 10(11 Suppl 18):6-9

31. McMillan LE, Brown JT, Henley JM, Cimarosti H (2011) Profiles of SUMO and ubiquitin conjugation in an Alzheimer's disease model. Neurosci Lett 502(3):201-208

32. Fukuda I, Ito A, Hirai G, Nishimura S, Kawasaki H, Saitoh H, Kimura K-I, Sodeoka M et al (2009) Ginkgolic acid inhibits protein SUMOylation by blocking formation of the E1-SUMO intermediate. Chem Biol 16(2):133-140

33. I. M. Janssen, S. Sturtz, G. Skipka, A. Zentner, M. Velasco Garrido, M. V Garrido, and MV. Garrido R. Busse, "Ginkgo biloba In Alzheimer's disease: a systematic review.," Wien Med Wochenschr, vol. 160, no. 21-22, pp. 539-546, 2010.

34. Sclip A, Antoniou X, Colombo A, Camici GG, Pozzi L, Cardinetti D, Feligioni M, Veglianese P et al (2011) C-Jun N-terminal kinase regulates soluble $\mathrm{A} \beta$ oligomers and cognitive impairment in $\mathrm{AD}$ mouse model. J Biol Chem 286(51):43871-43880

35. Mellone M, Stanic J, Hernandez LF, Iglesias E, Zianni E, Longhi A, Prigent A, Picconi B et al (2015) NMDA receptor GluN2A/GluN2B subunit ratio as synaptic trait of levodopa-induced dyskinesias: from experimental models to patients. Front Cell Neurosci 9:245

36. Hsiao K, Chapman P, Nilsen S, Eckman C, Harigaya Y, Younkin S, Yang F, Cole G (1996) Correlative memory deficits, Abeta elevation, and amyloid plaques in transgenic mice. Science 274(5284): 99-102

37. Puzzo D, Gulisano W, Palmeri A, Arancio O (2015) Rodent models for Alzheimer's disease drug discovery. Expert Opin Drug Discov 10(7):703-711

38. Grilli M, Summa M, Salamone A, Olivero G, Zappettini S, Di Prisco S, Feligioni M, Usai C et al (2012) In vitro exposure to nicotine induces endocytosis of presynaptic AMPA receptors modulating dopamine release in rat nucleus accumbens nerve terminals. Neuropharmacology 63(5):916-926

39. Pittaluga A, Feligioni M, Longordo F, Luccini E, Raiteri M (2006) Trafficking of presynaptic AMPA receptors mediating neurotransmitter release: neuronal selectivity and relationships with sensitivity to cyclothiazide. Neuropharmacology 50(3):286-296

40. Pittaluga A, Segantini D, Feligioni M, Raiteri M (2005) Extracellular protons differentially potentiate the responses of native AMPA receptor subtypes regulating neurotransmitter release. Br J Pharmacol 144(2):293-299

41. Lin TY, Lu CW, Huang SK, Wang SJ (2012) Curcumin inhibits glutamate release from rat prefrontal nerve endings by affecting vesicle mobilization. Int J Mol Sci 13(7):9097-9109

42. Tuz K, Peña-Segura C, Franco R, Pasantes-Morales H (2004) Depolarization, exocytosis and amino acid release evoked by hyposmolarity from cortical synaptosomes. Eur J Neurosci 19(4): 916-924

43. Becker-Hapak M, McAllister SS, Dowdy SF (2001) TAT-mediated protein transduction into mammalian cells. Methods 24(3):247-256 